\title{
Compliance with ecological safety requirements in air transport organizations
}

\author{
Lyudmila Kopteva ${ }^{1,2, *}$, Irina Romanova ${ }^{1}$, Maria Ivanova $^{3}$ and Vladimir Kulakov ${ }^{4}$ \\ ${ }^{1}$ Saint-Petrsburg State University of Aerospase Instrumentation, 67, Bolshaya Morskaia str., 190000, \\ Saint-Petersburg, Russia \\ ${ }^{2}$ St. Petersburg Branch Russian Customs Academy, Sofijskaya, 52, lit. A, 1912236, Saint-Petersburg, \\ Russia \\ ${ }^{3}$ Saint-Petrsburg State University of civil aviation, 38, ul. Pilots, 196210, Saint-Petersburg, Russia \\ ${ }^{4}$ Financial university under the Government of the Russian Federation, 49, Leningradsky Prospekt, \\ 125993, Moscow, Russia
}

\begin{abstract}
The relevance of the article is determined by the fact that air transport in general and the infrastructure of the airports in particular have strong negative impact on the environment. Some factors influencing the solution of this problem are considered in article, including the human factor. The quality management system in civil aviation organizations and the ecological strategy in the organizations of air transport are analysed. The examples of some Russian airports are studied. According to the authors, in addition to special technical measures, it is possible to use the incentive mechanism for employees of the air transport organization to form their responsible attitude to the accomplishment of their functional responsibilities regarding the potential impact on the ecological situation in the region.
\end{abstract}

\section{Introduction}

Aviation has a negative impact on the environment, especially on the areas located close to aerodromes and airport complexes. Aircraft noise, emission of harmful substances, acoustic impact, electromagnetic radiation - all these are negative impact factors. At present, new types of aircraft are being developed and produced, where maximum possible reduction of these factors is provided for. But, regretfully, the ecology of a modern airport often leaves much to be desired.

The state policy priority in the field of civil aviation (CA), according to ICAO (International Civil Aviation Organization) of which Russia is a member is governmental support of two aspects only: flight safety and aviation ecology. The state policy in the field of civil aviation facilities ecology is based on monitoring the environment in the course of facility operation and environmental protection measures, both preventive and protective. Today, the control of environmental status of civil aviation enterprises and facilities in Russia is exercised by the territorial bodies of the Federal Service for Environmental,

\footnotetext{
*Corresponding author: lusis63@mail.ru
} 
Technological, and Nuclear Supervision and the Federal Service for Consumer Rights Protection and Human Welfare [1,2].

The air transport organizations develop environmental strategies that must meet the requirements of the national regulatory documents and international standards.

However, the realization of the strategy is carried out at the level of linear management. Therefore, it is necessary to take the human factor into account and improve the employees' culture in the sphere of security. For these purposes, it is possible to use the incentives mechanisms for stimulating the air transport organizations employees, in order to form their responsible attitude towards fulfilment of their functional duties relating to possible impact on the ecological situation [3].

\section{Quality management system in civil aviation organizations}

Almost all federal aviation rules (FAR) contain requirements for development and realization of a quality management system (QMS) by civil aviation organizations whose activities are subject to mandatory certification. QMS is based on ISO 9000 requirements (ISO - International Organization for Standardization). The development and implementation of QMS that would comply with the ISO 9000 requirements is recommended for introduction only in organizations where this measure is a strategic decision of the top management. A civil aviation organization that has introduced and maintains QMS (regardless of whether a QMS certificate has been obtained) gains a competitive advantage. The availability of effective QMS creates a condition for integration of other management systems, in particular, the environment management system (EMS) in accordance with the requirements of certain standards (for instance, ISO 14000 series).

The ISO 14000 series of standards is based on voluntary approach to environmental regulation. The ISO 14001 standard establishes criteria for EMS. It does not set requirements for eco-efficiency, but describes the basic rules to be pursued by an organization to construct an efficient EMS. It can be used by organizations to improve resource efficiency use, to reduce losses and undue costs. Its principal objective is to help the companies to improve their environmental performance, while complying with the current legislation. Using ISO 14001, the company demonstrates the security of organization management and its employees.

In order for EMS to be validly accepted by managers of Russian enterprises and used in the most efficient way, the principles of extending EMS should primarily reflect the fact that this system acts as an effective tool for sustained business development.

Let us consider these principles in more detail.

1. The importance of environmental management approach for solving enterprises' internal problems. One of the main tasks is to demonstrate the possibility of real reduction of environmental impact by economically viable methods as a result of introducing EMS.

2. The importance of social aspects of environmental management. The concept of environmental management is based on the idea of interaction of an organization with the environment in which it operates. At the same time, consideration is given not only to the natural or exclusively economic environment (suppliers and consumers), but also to social environment. The latter is formed by interested parties representing different sectors of the society. The minimum scope of interaction with interested parties is set by EMS standards. Thus, according to ISO 14001, it is necessary to develop and ensure accessibility of enterprises' environmental policy.

In recent years, increased interest of enterprises, including air transport organizations, in realization and certification of EMS in accordance with ISO 14001 series standard has been observed. This is due to the increased environmental awareness of the public and consumers and the growing struggle between the enterprises for obtaining the 
"environmentally friendly" status. An important step in formation of EMS is development of environmental strategy setting the general direction of its operation in the environmental field [4].

\section{Results}

The environmental strategy of the enterprise provides for the development of integrated measures towards solution of environmental problems aimed at:

- rational use of resources;

- curtailment of emission (preventing or reducing the release of harmful substances into the environment);

- waste reduction (reduction of the, recycling and reuse);

- production of harmless products that do not cause harm throughout the life cycle in terms of emission and waste and are characterised by low risk.

The choice of environmental strategy determines the formation of due environmental policy of the enterprise, which represents a set of publicly declared principles and commitments related to the ecological aspects of operation of the enterprise and providing a basis for establishment and achievement of its environmental goals and objectives. The development of environmental policy is one of the primary and most crucial steps in inculcation of EMS at the enterprise. The ISO 14001 standard sets out the basic requirements to the contents of environmental policy; it states as well that the top management is in charge of the development and adoption of the environmental policy.

As an example, let us cite the provisions of the environmental policy of Northern Capital Gateway, Ltd. (NCG), Pulkovo Airport operator, that occupies one of the leading positions in the market of aviation transport services in the Russian Federation.

Being aware of its responsibility for preservation of the environment in the process of its activities, NCG management assumes the following obligations:

- to carry out the activities in accordance with the current legislation and other environmental requirements applicable to NCG's activities;

- to make ecological monitoring of its activities and of their impact on the environment;

- to explore the possibilities of reducing the environmental impact of various aspects of production;

- to introduce modern, efficient methods and technologies in the modernization of existing production processes, seeking to reduce the environmental stress;

- to minimize any unpredictable environmental impact in daily activities by developing due measures to prevent accidents, and their timely realization;

- to develop an environmental management system, to take managerial decisions based on the analysis of its activities in respect of the environment;

- to seek to establish a productive dialogue on environmental issues with interested parties $[5,6]$.

The organizational basis for the realization of these commitments is the environmental management system. This policy is an integral part of the business strategy of NCG which regularly monitors its environmental impact on the air, water and soil.

NCG has developed a concept for monitoring noise impact. In particular, it engages in measurement of noise impact caused by aircraft. NCG interacts with a number of airlines to reduce the aircraft noise impact and to reduce the greenhouse gas emission.

The noise impact reduction measures include optimization of the take-off and landing pattern according to the flight instruction; recommendations for heightening the vectormode landing approach phase; gradual withdrawal of outdated and noisy aircraft models from the airline fleet, such as Tu-134, Tu-154B, Tu-154M, Yak-42 and Il-76; 
recommendations for preferred use of a particular runway, located further from residential areas, at night (from 23:00 to 7:00).

The measures to reduce greenhouse gas emission include providing ground-based electric energy to airlines' aircraft, when parking, in order to avoid their use of the auxiliary power unit for power consumption. Currently, this service is provided at airline's request.

As part of the renovation of the airport, the design of stormwater runoff treatment facilities and the development of amelioration and drainage system have been undertaken.

Waste management system has been developed and implemented, involving separate waste collection, both in air terminal buildings and enterprise units; with minimizing waste generation and increasing the generation of recoverable resources [7].

The fleet of ground service vehicles has been upgraded (with acquisition of combinedpurpose transport for conveying luggage and electromobiles, where appropriate).

A project of sanitary protection zone (SPZ) and a sanitary gap has been launched for protection of land and houses, to cover $28.5 \mathrm{~km} 2$ around the airport complex, with 34 houses and 36 land plots that are subject to "security and protection".

In addition to the above activities exercised by Pulkovo Airport within the framework of the environmental policy, it is necessary to dwell on another external factor that cannot be ignored in developing the ecological strategy. The urban development is closely approaching Pulkovo airport, so it has nowhere to extend when such a need arises. Several hypermarkets are now being erected right under the glide path. This project raises many questions connected with both flight safety and aviation ecology. Aeroplanes emit harmful residues of combustion products; they might discharge them, flying at low altitude over the stores; in addition, they can discharge fuel before landing [8].

In the Russian Federation, the methodological approaches, methods and programmes applied for calculating the level of aircraft noise and concentration of pollutants in the atmospheric air caused by aircraft engines are incoherent. This is particularly evident today in development of SPZ projects at CA airports. This circumstance leads to significant errors in outlining the boundaries of airports' SPZ, which in some cases hinders the development of the latter and causes objective difficulties for sanitary-hygienic examination of the projects.

The lack of a uniform procedure for development of projects and mechanisms for verifying proposed SPZ design boundaries and airport sanitary gaps, as well as the absence of a unified up-to-date methodological basis for assessing the specific impact of airborne vehicles on the environment, not only constrains the development of projects, affecting their quality and disrupting the timeframe of project expert examination, but also builds a hindrance to adequate account of the functional use of sanitary gap zones in the spatial planning of aerodrome environs [9].

Among the socio-psychological qualities affecting safety, motivation ranks first. It is known that ignoring safety standards entails damage caused to the employees, the society, the enterprise, the ecology.

In order to involve a person in the solution of a particular task, it is necessary to find motivation that would induce him to act. The positive motivation aims at achieving success in one's activities and supposes conscious activity; it is connected in a certain way with manifestation of positive emotions and feelings. Meanwhile the negative motivation - like disapproval, dispraise - entails punishment not only in material aspect, but also in psychological context, as a rule. With negative motivation, a person seeks to escape failure. The fear of negative reward usually leads to emergence of negative emotions and feelings, and, as a consequence, may result in violation of safety rules and grave consequences, especially in the aviation industry.

Therefore, it is necessary to take into account that repeated use of penalty significantly reduces its effect: a person becomes accustomed to the negative impact of punishment and 
eventually stops responding to it. Consequently, the long-lasting negative motivation adversely affects not only the labour results, but also the employee's personality.

Under the influence of motivation ("re-motivation"), the adequacy of one's perception of a real situation may be disrupted, and an undesirable event (for example, a dangerous routine or emergency situation) may seem less likely. On the other hand, the stronger the motive to achieve a goal that is significant for a person, the greater the threat of its nonachievement. The situation here is subject to a pattern established by American psychologists R. Yerkes and J. Dodson on the basis of experimental studies, which has become known as Yerkes-Dodson's law: "An activity does not yield success when a person does not want something or wants it excessively".

Let us consider the motives of industrial labour and their relationship. According to the research, labour encompasses five main motives: benefit, safety, convenience, satisfaction, fitting into the work collective.

The motive of benefit lies in receiving remuneration for the results of labour. The concept of benefit includes material benefit (salary, bonuses) and social benefit (prestige, self-affirmation, professional pride).

The motive of safety reflects the desire to avoid any dangers arising in the labour process, as well as confidence in the future.

The motive of convenience is based on the desire to choose the easiest way of performing the task, requiring less energy, less mental stress.

The motive of satisfaction means to enjoy the labour process and feel satisfaction with the result.

The motive of fitting into the team is manifested by the urge to act in accordance with the way customary for a particular work group.

All of the above motives are present in the activities of any employee. However, the role and significance of a particular motive is not the same for different employees in terms of overall motivation. The hierarchy of motives, along with the hierarchy of needs, reflects the core of a personality which can be defined as one's orientation.

It is possible to identify some objective factors that affect the extent of motivation towards performance of functional duties, connected with observance of safety in the professional activities of civil aviation workers.

1. The effect of awareness of the motivation object. If an air transport organization employee does not clearly realize the hazards associated with his/her labour, and therefore, does not realize sufficiently the need for protective equipment and safety regulations, then the strength of motivation to use these means and follow the rules will be low.

2. The effect of skills on the extent of motivation. Skills contribute to improved performance, and more efficient work becomes more attractive, following its implementation. Consequently, by developing safety compliance skills, aviation specialists enhance their own motivation to follow these rules.

3. The effect of initial attractiveness of motivation objects on the power of motives. It is common for man to idealize attractive objects, which increases further aspiration for these objects or makes them an object of other motives.

4. The effect of difficult nature of a task on the strength of motivation towards its implementation. A motive has the greatest strength in performing tasks of medium complexity - in this case the grounds to succeed are sufficient, still there are some difficulties making success even more attractive, when overcome.

The motives are often contradictory, leading to a conflict. Let us analyze a possible conflict between the benefit and safety motives.

If we formally assess the impact of various rules and safety instructions on the labour process, it is easy to notice that all of them, protecting a worker from hazards, simultaneously impose appropriate restrictions on his behaviour. Without these rules, the 
worker could perform his actions in any way that he would deem beneficial, convenient for himself at that moment. The rules set some limitations within which he is supposed to work. Similarly, environmental protection means protect the objects of nature located in environmental protection zones of airports and airport complexes from danger, at the same time restricting to some extent the freedom of actions of air transport organizations personnel.

There is a concept of the so-called "goal gradient" in psychology, according to which the motive to achieve a purpose gets intensified to the extent the goal is approaching; or, in other words, in case of rivalrous motives of equal strength, the one that is realized faster wins (it has a higher "gradient"). For a worker, the gain (greater output, faster accomplishment of a task, less effort in the production process, etc.) is realized on the spot, in the process of work, so the probability of benefit is higher than the probability of threat. That is why the "gradient" of gain is higher than the "gradient" of danger, and this contributes to the choice of behavioural strategy oriented towards reaping the benefit.

The strength of the gain motive (attainment of a real goal) is growing pro rata approaching it. Meanwhile the strength of the safety motive (avoidance of possible danger) is growing insignificantly as it approaches the goal, but it increases dramatically on the threshold of reaching the goal, when it is close and its realization seems possible, against the background of some danger menacing it. Though, if the realization of threat is regarded as unlikely event, then the safety motive may remain at a low level and not increase at all.

It follows from the above dependencies that there are some objective reasons that make the motive of gain more preferable than the safety motive. This entails some possible ways of coping with this unfavourable situation:

1. To find the ways to confirm and strengthen the safety motive, i.e. widespread use of incentives for safe work. It is necessary to stimulate safe work in the same way as efficient output - materially and socially. If we consider the total losses caused by accidents, we might find that the expenditure on such stimulation is recouped multiply by reducing losses from injuries.

2. Creation of such labour conditions and technical facilities that would construct a situation when obtaining benefit from violating the rules would be impossible. In case such facilities have not yet been introduced, it is necessary to cultivate the psychological climate in the collective team where losing one's credibility because of violation of safety rules would negate all material benefits that could have been achieved through violations.

Self-actualization, responsible attitude to one's duties, adherence to labour and technological discipline should become a motivational dominant in aviation personnel's professional activity. Nowadays, young people entering the aviation labour market after receiving an appropriate education often practice the behaviour based on consumer demands. Their motivational dominant is the material component, which is manifested in expectation of high salary in the industry. The presence of this dominant makes it possible to suppose the onset of the so-called "motivation crisis" which is unacceptable in the aviation industry where the work involves high responsibility and provision of security. To overcome this problem, it is necessary to focus on the use of such powerful resource as counselling and coaching as a competent tool for the manager. Trust and support are powerful motivators involving the desire to take responsibility for results and to make one's best to achieve them. An important psychological effect lies in one's permanent search for an example, reference to be used a basis for solving problems, including those relating to safety. It is necessary to find an approach to formation of professional motivation that would let one unlock his/her intellectual and creative potential to the uttermost, stimulate the interest of the aviation personnel in their profession, create a basis for cultivating corporate identity. 
The use of modern equipment and technologies, the availability of physical infrastructure adequate to the tasks of the enterprise cannot guarantee efficient and troublefree operation of air transport organizations if the said technical facilities are used and operated by insufficiently qualified and motivated specialists.

The human factor is undoubtedly a key element of the ergonomic system "man aircraft - airfield - environment" which conditions the extent of reliability and durability of airfield facilities, safety and regularity of aircraft flights.

\section{Conclusions}

When developing and implementing the environmental strategy for airports, it is necessary to take into account the human factor and staff motivation towards compliance with all regulations and standards in the sphere of environmental and operational safety. As part of the quality management system, it is possible to use environmental management approaches for the solution of internal problems of enterprises and to ensure interaction of the air transport organization with the environment in which it operates. At the same time, it is necessary to pay due attention to the natural environment, as well as economic and social environment. In particular, it is possible to develop a staff motivation system under which the employee would be interested in his/her work and in the efficient operation of the enterprise in general, which will help to improve flight safety, passenger service culture and competitiveness of the company within the country and in the international market of transport services. In our opinion, in the down economy, it is necessary to make a focus on intangible incentives, since this does not require substantial material investments, but still allows a company to achieve the goals involving efficient use of its human resources.

\section{References}

1. V.G. Bogdanov, M.O. Ivanova, N.A. Petriy, Materials of IX International Academic Workshop (Centre of Professional Management - Academy of Business Publishing House, Saratov, 2015)

2. M.O. Ivanova, Materials of X Annual Ecological Academic Youth Conference 1, 39-45 (2016)

3. A. Seleznev, A. Mottaeva, L. Andreeva, S. Izmaylova, IOP Conf. Series: Earth and Environmental Science 90, 012117 (2017) doi: 10.1088/1755-1315/90/1/012117

4. E. Mitrofanova, L. Alshanskaya, HR Pro 4, 61-69 (2015)

5. R. Yerkes, J. Dodson, J. Comp. Neurol. Psychol 18, 459-482 (1908)

6. A. Mottaeva, E. Vasilyeva, MATEC Web of Conferences 239, 04019 (2018) doi.org/10.1051/matecconf/201823904019

7. G. Dyakova, S. Izmaylova, A. Mottaeva, E. Karanina, IOP Conf. Series: Earth and Environmental Science 90, 012218 (2017) doi :10.1088/1755-1315/90/1/012218

8. E. Vasilyeva, I. Polyakova, MATEC Web of Conferences 239, 08018 (2018)

9. M.O. Ivanova, V.N. Ivanchenko, Proceedings of XIX All-Russian Academic Workshop. St. Petersburg 1, 159-162 (2016) 